\title{
Inhalt des 123. Bandes.
}

\author{
Erstes Heft (2. Januar).
}

I. Zur Leichendiagnose der septischen und pyămischen Prozesse. (Aus dem Pathologischen Institut zu Berlin.) Von Dr. med. Martin Hahn. . . . . . . . . . . . . . . . . .

II. Ueber den intermediären Kreislauf des Fettes durçh die Leber, seine physiologische Bedeutung und seine Beziebungen zum Icterus neonatorum. Aus dem thierphysiologischen Laboratorium der kgl. landwirthschaftlichen Hochschule zu Berlin. Von Dr. Siegfried Rosenberg in Berlin. . . . . . . . . . .

III. Die Circulation im Gehirn und ihre Störungen. III. Vertheidigung der Grundgesetze. Vom Privatdocenten Dr. Richard Geigel in Würzburg. . . . . . . . . . . . . . . . . .

IV. Ueber das Auftreten von Oxyhämoglobin in der Galle. (Aus dem Laboratorium der medicinischen Klinik des Herrn Gebeimrath Biermer in Breslau.) Von Dr. Richard Stern, Assistenten der Klinik. . . . . . . . . . . . . . . .

v. Ueber das Brechcentrum und über die Wirkung einiger pharmakologischer Mittel auf dasselbe. Von Prof. Lazarus J. Thumas in Warschau. . . . . . . . . . . . . . . . . . .

VI. Ein Fall von septischer Pneumonie heim Neugebornen, verursacht durch den Bacillus enteritidis (Gaertner). Von Dr. 0. Lubarsch, Privatdocenten und Assistenten am Patbologischen Institut in Zürich, unter theilweiser Mitarbeiterschaft von Dr. H. Tsutsui aus Tokio. (Hierzu Taf. I und 1 Zinkograpbie.).

VII. Ein Fall von Lymphangioma tuberosum multiplex (Kaposi). Von Dr. E. Lesser und Dr. R. Beneke in Leipzig. (Hierzu Taf. II. Fig. 1-2.) . . . . . . . . . . . . . . . . VIII. Beitrăge zur Anatomie der ektopischen Schøangerschaften. (Aus dem pathologischen Cabinet des Peter-Paul-Hospitals in St. Petersburg.) Von Dr. Th. Dobbert. (Hierzu Taf. II. Fig. 3-4.) 103 
IX. Ein Fall von angeborner Hypertrophie des Herzens. Von Dr. Guido Rbeiner in St. Gallen. . . . . . . . . . . .

X. Arbeiten aus dem pharmakologischen Institut der Universität Breslau. Experimentaluntersuchungen über Antipyrese und Pyrese, nervöse und künstlicbe Hypertbermie. Von Dr. Paul Richter, prakt. Arzt. (Mit 5 Holzscbnitten.) . . . . . .

XI. Polynenritis recurrens. (Aus der medicinischen Klinik des Herrn Prof. Eichhorst in Zürich.) Von Mary Sberwood aus New York U. S. Am. (Mit 1 Holnschnitt.) . . . . . . . . . 166

XII. Kleinere Mittheilungen.

1. Zur Casuistik des chylösen Ascites. Aus dem Laboratorium der medicinischen Klinik in Göttingen. Von Dr. med. Hans Reichenbach. . . . . . . . . . . .

2. Der intermediäre Kreislauf des Fettes durch Leber und Gallenblase. Von Rud. Virchow. . . . . . .

3: Ueber die Höhe und die Höhenzahl des Gewichts und des Volumens von Menschen und Thieren. Vorlãufige Mittheilung. Von Dr. Mies, z. Z. in Berlin. . . . . . 188

XIII. Besprechung. .

Die Grippe-Epidemie im Deutschen Heere 1889/90, besprochen von Prof. Mosler in Greifswald. . . . . . . . . . 193

\section{Zweites Heft (3. Februar).}

XIV, Mittheilungen aus dem pathologisch-anatomischen Institut zu Genf. Von Prof. F. Wilh. Zabn.

1. Ueber die Entstehungsweise von Pneumothorax durch Continuitātstrennung der Lungenpleura ohne eitrige Entzündung. . . . . . . . . . . . . . . . . .

2. Ueber einen Fall von ulceröser Entzündung der Trachea und des linken Bronchus in Folge eines Aneurysma des Aortenbogen's mit Durchbrucb in die Trachea. . . . .

XV. Zur Lebre von der Resorption im Darm, nach Untersuchungen an einer Lymph(chylus-)fistel beim Menschen. Von Immanuel Munk und A. Rosenstein in Berlin. (Hierzu Taf. III.)

XVI. Beitrăge zur Pathologie der Kakke. Von Dr. M. Miura in Tokio, Japan. (Hierzu Taf. IV-VI.) . . . . . . . . . 280

$X$ VII. Ueber den Nachweis und die quantitative Bestimmung der Salzsăure im Magensaft. (Aus dem chemiscben Laboratorium des Pathologischen Instituts zu Berlin.) Von Dr. A. Fawizky aus Petersburg. . . . . . . . . . . . . . . . . .

XVIII. Die anāmiscbe Nekrose der Nierenepithelien. Experimentelle Untersuchung. Von Dr. Oscar Israel, Privatdocenten und 
I. anatomischem Assistenten am Pathologischen Institut zu Berlin. (Hierzu Taf. VII, VIII, VIII a, IX.) . . . . . . . . . . 310

XIX. Ueber die nach Lösung incarcerirter Ilernien auftretenden Lungenerscheinungen. (Aus dem Pathologischen Institut in Berlin.) Von Dr. Johannes Lesshafft. . . . . . . . . . 335

XX. Ueber patbologische Mitosen. Von Dr. I) avid Hansemann, Assistenten am Patbologiscben Institut und Privatdocenten an der Universität zu Berlin. (Hierzu Taf. X-XI.) . . . . . 356

XXI. Kleinere Mittheilungen.

1. Ein seltener Fall von Brücken-Colobom der Iris. Von Dr. Richard Hilbert in Sensburg. (Mit 1 Zinkographie.)

2. Ueber die künstliche Darstellung von barnsauren Salzen in der Form von Sphärolitben. Aus der medicinischen Universitätsklinik zu Göttingen. Von Wilhelm Ebstein und Arthar Nicolaier. (Mit 1 Zinkographie.) . .

3. Vorläufige Mittheilung über eine bakteriolog.-experiment. Untersuchung zur Frage der Puerperaleklanpsie. Aus dem Pathologiscben Institut in Berlin. Von Dr. Alexandre Favre.

4. Medicinisch-naturwissenschaftlicher Nekrolog des Jahres 1890, zusammengestellt von Dr. med. Wilh. Stricker, prakt. Arzte in Frankfurt a. M. . . . . . . . . . 378

5. Berichtigungen. Von Wilh. Filebne. . . . . . 388

\section{Drittes Heft (4. März).}

XXII. Ueber das Vorbandensein von Gescbmacksempfindung im Kehlkopf. (Ausgearbeitet nach einem am 1. December 1890 in der biologischen Gesellschaft zu Königsberg gehaltenen Vortrag.) Von Dr. P. Michelson, Privatdocenten in $\mathbf{K o ̈}-$ nigsberg. . . . . . . . . . . . . . . . . . .

XXIII. Beiträge zur Kenntniss der Missbildungen in der Kopf - und Halsgegend. Von K. v. Kostanecki, Volontär-Assistenten am I. anatomischen Institut in Berlin. (Bierzu Taf. XII.) . 401

XXIV. Beitrag zur Kenntniss des Glioms der Netzhaut. Von Dr. W. Eisenlohr in Freiburg i. B. (Hierzu Taf. XIII.) . .

XXV. Ein Fall von multiplen Cystadenomen der Gallengänge mit Durchbruch in's Gefässsystem. Von Dr. E. von Hippel, II. Assistenten am pathologischen Institut $2 \mathrm{u}$ Heidelberg. (Hierzu Taf. XIV.) . . . . . . . . . . . . . . .

XXVI. Zur Lehre von der Resorption im Darm, nach Untersuchungen an einer Lymph(chylus-)fistel beim Menschen. Von I m manuel Munk und A. Rosenstein in Berlin. (Schluss von S. 279.) \$\$4 
XXVII. Ueber das Indigoroth (Indirubin). Aus der medicin. Abtheilung des Herrn Prof. O. Rosen bach im Allerbeiligen-Hospital zu Breslau. Von Dr. Heinrich Rosin, Assistenzarzt. .

Seite Ueber die angeblicbe Bedeutung intravasculärer Gerinnungen als Todesursache bei Vergiftungen durch Anilin, chlorsaure Salze und Sublimat. Aus dem Pathologischen Institut zu Marburg." Von Dr. Wilhelm Falkenberg. . . . . . 567

Nachtrag von Prof. Marchand. . . . . . . . . . 587

XXIX. Arbeiten aus dem pharmakologiscben Institut der Universität Breslau. Experimentaluntersuchungen über die Darmwirkung des Opiums und Morpbins. Von der medicinischen Facultăt zu Breslau gekrönte Preisscbrift. Von Wilhelm Spitzer, prakt. Arzt. . . . . . . . . . . . . . . . . . 593

XXX. Kleinere Mittheilungen.

1. Weitere vorlăufige Mittheilung über Puerperaleklampsie mit Berücksichtigung der dabei vorkommenden Erosiones baemorrbagicae ventriculi. (Aus dem Pathologischen Institut in Berlin.) Von Dr. Alexandre Favre. . . . 628

2. Die Regulirung der Blutbewegung in Gehirn. Von Dr. Benno Lewy in Berlin. 